\title{
The Prevalence of Smoking and its Impact on Disability in Multiple Sclerosis
}

\author{
Daliborka Tadić1,2, Vlado Đajićn ${ }^{1,2}$, Sanja Grgić1,2, Siniša Miljković1,2
}

\begin{abstract}
Introduction: Etiology of multiple sclerosis (MS) involves multi-factorial interactions among genetic and environmental factors. Cigarette smoking is one of the most important environmental factors that increase the disease risk by about $50 \%$. The aim of this research was to assess the prevalence of smoking in MS patients compared to the general population, and to assess the association between smoking and physical disability in MS patients.

Materials and Methods: A cross-sectional study included 100 MS patients and 50 healthy people. For estimation of the degree of physical disability, the Expanded Disability Status Scale (EDSS) was used, and for clinical and demographic data a general questionnaire was used. In order to collect data on the smoking, the questionnaire for examining risk factors for vascular disease among patients with MS was used.

Results: Analysis of smoking incidence showed that, between MS and control group, smoking was a dependent variable $\left(\chi^{2}=6.258, p=0.04\right)$. There was no statistically significant difference in the number of cigarettes, nor in the duration of smoking. There was no significant correlation between smoking in MS group and the index of disease progression $(\mathrm{r}=0.216, \mathrm{p}=0.133)$. Also, the relationship between EDSS and smoking had no statistical significance, as well as between the disease course and smoking $\left(\chi^{2}=1.531, \mathrm{p}=0.216\right)$.

Conclusion: Although it seems reasonable to restrict or refrain from smoking in patients with MS there was no significant evidence of smoking and disease progression correlation.
\end{abstract}

Key words: multiple sclerosis, smoking
(1) Clinic of Neurology, University Clinical Center of the Republic of Srpska, Banja Luka, Republic of Srpska, Bosnia and Herzegovina

(2) Faculty of Medicine, University of Banja Luka, Republic of Srpska, Bosnia and Herzegovina

\section{INTRODUCTION}

Multiple sclerosis (MS) is a chronic, autoimmune, inflammatory, demyelinating disease of the central nervous system and one of the most common causes of disability in the category of younger adults. ${ }^{1}$ The disease has a very heterogeneous clinical course and symptoms. Since the average duration of the disease is longer than twenty years, the accumulation of clinical manifestations may significantly affect the daily living of patients. ${ }^{2}$ The etiology of MS involves multifactorial interactions among genetic and environmental factors. Genetic predisposition is only a part of the risk for developing MS, while lifestyle and environmental factors are key participants in the development of the disease. The influence of these factors on the pathogenetic mechanisms is very important and some of them can be modified. Proven risk factors for developing the disease are female gender, smoking, low levels of vitamin D, dietary habits, Epstein Barr virus infections and obesity in childhood and adolescence. ${ }^{3}$

Cigarette smoking is, however, one of the most important environmental factors that increase the risk of disease by about 50\%. These are data from previously performed studies that indicate 
that the precise effects of smoking on the onset and development of MS vary in different contexts and different populations. ${ }^{4}$ Since the '6os of the last century, smoking has been identified as a significant risk factor for the occurrence and progression of MS. Israeli researchers found a greater percentage of smokers among MS patients than in the general population (44: $36 \%) .{ }^{5}$ Several other studies based on same questionnaire also confiremd the above findings. ${ }^{6,7}$

Recently, considerable research was focused the nicotine metabolite (cotinine) in the blood, and it was shown that nicotine alone was less important for the onset and progression of the disease than the non-nicotine substances produced by the combustion of tobacco. Most of these studies were related to patients tested after the diagnosis of MS. ${ }^{8-10}$ A prospective study by Salzer et al. confirmed that smoking was as a risk factor for MS; the risk for MS was increased by $50 \%$ in patients with elevated levels of cotinine in the blood. ${ }^{11}$

According to the study of Hedstrom et al, patients who were smokers and treated with interferon beta $1 b$ therapy had an increased risk of the development of neutralizing antibodies to the drug in comparison to the non-smokers. Such a correlation was not observed in former smokers. ${ }^{12}$ Olsson and co-workers showed that smoking had a significant impact on the occurrence of neutralizing antibodies to natalizumab, which reduces or disables the therapeutic effect of this drug. It was also shown that smoking is associated with a greater number of relapses in natalizumab-treated MS who were smokers compared to those who did not smoke. ${ }^{13}$ The effect of smoking on the presence of antibodies against John Chunningam (JC) virus in the population of MS patients was also studied, and it was established that smoking is not associated with an increased prevalence of the presence of this virus. ${ }^{14}$

\section{AIM OF THE STUDY}

The aim of this study was to assess the frequency of cigarette smoking in a population of MS patients, compared to the general population matched by gender and age, as well as to estimate the potential connection of smoking and physical disability measured by The Expanded Disability Status Scale (EDSS) in patients suffering from MS. ${ }^{15}$

\section{METHODS}

This cross-sectional study was conducted as part of the examination of patients affected by MS in ambulances or during hospitalization at the Department of Neurology, University Clinical Center of the Republic of Srpska, Banja Luka. The sample consisted of 100 MS patients (MS group) and 50 healthy people (control group). The inclusion criteria were: MS disgnosed according to the McDonald criteria, patients age 18-69 years, and consent to participate. ${ }^{3}$ The exclusion criteria were: An exacerbation of the last month, and the use of corticosteroids of the last month.

The study was conducted for twelve months. Participants, both MS patients and healthy people, were introduced with the basic elements of research and voluntarily signed the consent for participation in the study. The study was approved by the Ethics Committee of University Clinical Center of the Republic of Srpska, Banja Luka, Republic of Srpska, Bosnia and Herzegovina. A general questionnaire, consisted of questions related to the clinical characteristics of patients: age at onset of MS, duration of illness, the course of MS and value of EDSS score at the time of testing was used. In order to determine the degree of physical disability, the Expanded Disability status scale (EDSS) was used..$^{15}$ In order to collect data on risk factors, it the questionnaire for risk factors for vascular diseases in patients with MS was used. The questionnaire was based on the literature data for the purpose of scientific research at the Institute of Epidemiology, School of Medicine, University of Belgrade. Data were related to the smoking status of participants (yes/no, ex-smoker), the number of cigarettes smoked per day and the duration of smoking habit (from- to).

Statistical analyses included methods of descriptive statistics (mean, standard deviation, mode, median, skewness/kurtosis), $\chi^{2}$ test, Student t-test, variance analysis (ANOVA), correlations (Pearson's and Spearman's tests) and regression analysis. 


\section{RESULTS}

Regarding clinical data relevant to this study, it is important that when it comes to the course of MS, the majority of our patients (72\%) had a relapsing-remitting form of $\mathrm{MS}, 27 \%$ had a secondary progressive MS, and $1 \%$ had primary progressive MS. The average value of EDSS score in MS group was $3.7 \pm 2.1$. The mean age of study subjects at enrolment was $41.9 \pm 10.1$ (range 2064 ) in MS group, and 42.1 \pm 12.3 (range 18-63) in control group. Out of the 100 subjects in MS group, 25 were male and 75 female, and out of 5 o subjects in control group 14 (28\%) were male and $36(72 \%)$ were female.

A detailed analysis of the frequency of smoking in studied groups showed that smoking was a dependent variable $(\chi 2=6.258, p=0.04)$. Figure 1 shows that the structure of the response varied between the MS and control group.

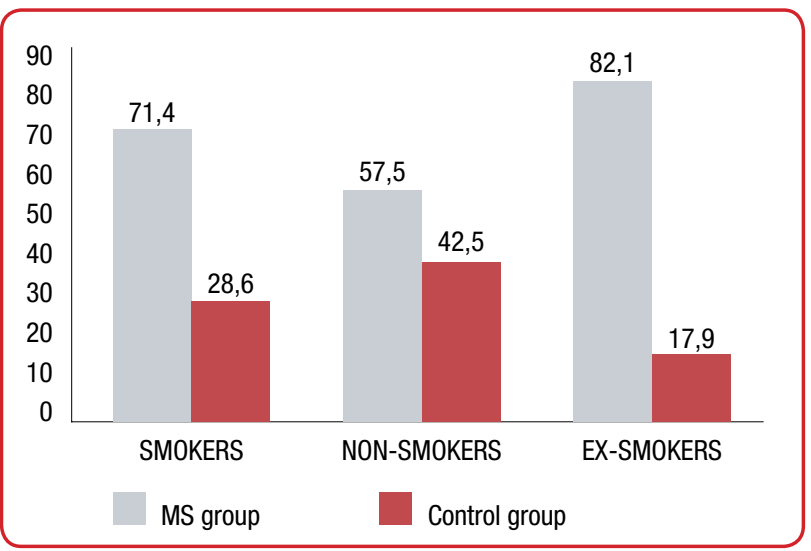

Figure 1: Representation of smoking in the control and MS group (\%)

Among smokers, analyzed in both groups, there was no statistically significant difference in the number of smoked cigarettes nor the duration of smoking (table 1).

Table 1: Representation of smoking in the control and experimental group (\%)

\begin{tabular}{|c|c|c|c|c|c|}
\hline \multirow[b]{2}{*}{ Group } & \multirow[b]{2}{*}{ Number } & \multicolumn{2}{|c|}{ Number of cigarettes } & \multicolumn{2}{|c|}{ Duration of smoking (years) } \\
\hline & & $\begin{array}{l}\text { Average } \\
\text { value }\end{array}$ & $\begin{array}{l}\text { Standard } \\
\text { deviation }\end{array}$ & $\begin{array}{l}\text { Average } \\
\text { value }\end{array}$ & $\begin{array}{l}\text { Standard } \\
\text { deviation }\end{array}$ \\
\hline MS & 58 & 15.17 & 8.10 & 18.47 & 10.54 \\
\hline Control & 19 & 16.12 & 6.77 & 23.16 & 10.18 \\
\hline
\end{tabular}

In terms of connection between smoking in the MS group and index of disease progression, the Pearson correlation coefficient was used. However, in relation to the analyzed variable, the correlation coefficient had a low absolute value, which means that there was no statistically significant correlation with an index of disease progression $(\mathrm{r}=0.167, \mathrm{p}=0.251)$. The Spearman correlation coefficient showed that the relationship between EDSS and smoking had no statistical significance.

In order to establish a possible relationship between the disease and smoking the chi-square test was used, but no significance was found ( $\chi^{2}$ $=1.531, \mathrm{p}=0.216$ ).

\section{DISCUSION}

A significant number of studies showed that smoking significantly increased, by $50 \%$, the risk for developing of MS. $5,6,8,16-19$

Results of this study showed that smoking, was a dependent variable $(p=0.04)$. Data from this survey also showed that the proportion of smokers in MS groups was statistically significantly different, as confirmed by the chi-square test. There were $71.4 \%$ of smokers in the MS group and $28.6 \%$ in the control group. Specifically, while in the experimental group share of smokers and ex-smokers dominates, the same share does not apply to the control group. Pekmezović and co-workers conducted a survey in the Belgrade area and showed that smoking is significantly more frequent in the group of patients with MS than in the control group. They also found that smoking was a significant and independent risk factor for MS. ${ }^{20}$ This was also shown in two studies by Hedstrom et al, where smoking was identified as a risk factor for the development of MS. ${ }^{21,22}$

Salzer et al. found that patients with higher risk were young adults up to 26.4 years of age. ${ }^{11}$ The same researchers evaluated the risk of passive smoking exposure on the occurence of MS and concluded that the risk was significantly higher in patients who were exposed to inhalation of tobacco smoke than those who were not. This risk increased with longer exposure to the 
smoke. ${ }^{23}$ They also analyzed blood samples in women who smoked during the gestation period and did not observe a higher risk of MS after childbirth, nor after 26.4 years of age of children whose mothers smoked during pregnancy. A weak link between smoking during pregnancy and the postpartal onset of MS was also shown by Montgomery and al. ${ }^{24}$ In a study published in 2007 it was shown that children whose parents smoke have a greater risk of developing MS, and this risk increased with higher exposure to passive smoking. ${ }^{25}$ Another research showed that there was a significantly higher risk for the development of MS in children who had a clinically isolated syndrome, and who were exposed to passive smoking compared to those who were not. ${ }^{26}$ Similar results related to young adulthood and exposure to risk factors (elevated BMI, cytomegalovirus infection, the level of vitamin D), and smoking as the most important risk factor for the appearance of the MS in that age were provided by other researchers. ${ }^{27,28,29}$

The results of our study showed that there was no statistically significant difference in the number of cigarettes smoked, nor in the duration of smoking. However, Iranian researchers in a study published in 2013 in a population of patients with MS established that the risk of MS was significantly higher in male smokers and in those who were taking of large amounts of cigarettes. $^{30}$ The same year, the results of another study showed the similar results as the above-mentioned one, with addition that this group of researchers found a statistically significant positive correlation between the number of cigarettes (more than ten) during the day, and the development of secondary progressive MS. ${ }^{31}$

In our study, we did not find correlation between the disease and smoking, nor the impact of smoking on the index of the disease progression. This can be explained by a certain specificity of our participants who were under regular medical control and without any significant influence of other comorbidity conditions. The high percentage of participants of the MS group (82.10\%) were former smokers, in contrast to the control group where the percentage was $17.90 \%$. The study of Hernan et al. showed that smokers with the relapsing-remitting (RR) form of MS are three times more likely to move to secondary progression than non-smokers, as only 20 of the 179 patients who had never smoked or were former smokers had a progressive course of the disease. ${ }^{7}$ In the study of Sundstrom et al, out of 122 patients with newly diagnosed MS, 72\% of them were ex-smokers, and those who started smoking before the age of 15 developed the secondary form of the disease after an average of six years of follow up. Forty percent of ex-smokers who started smoking after the age of 15 years developed a secondary progression, and it happened with only $26 \%$ of non-smokers. ${ }^{8}$

Di Pauli and associates observed 129 patients with the clinically isolated syndrome (CIS) who were at high risk for the MS according to the MRI and cerebrospinal fluid analyses. After the period of three years, $75 \%$ of smokers developed MS, compared to $51 \%$ of non-smokers. ${ }^{32}$

In contrast to these results, Seine et al. examined 205 women with clinically definite MS from Portugal and noted that smoking had a protective effect on women with ApoE4 allele. Smokers had lower scores on the EDSS and MSSS scale. ${ }^{33}$ Only one study, by Pittas et al, took into account the potential confounding effect of other habits of people with disease. ${ }^{34}$

In several studies, cigarette smoking is shown to be a factor associated with an increased level of gadolinium discolored lesions, and the volume of T2 hyperintense lesions on MRI, and brain atrophy. However, none of these studies had a control group of people without MS, which would give more informative results. ${ }^{35,}{ }^{36}$ Several other studies have shown a positive correlation between cigarette smoking and disease progression. ${ }^{37}$ Manouchehrinia et al. analyzed data from 895 patients, where $49 \%$ were smokers at the time of onset of the disease and achieving the diagnosis. Their results showed that smoking was associated with increased severity of the disease and more rapid progression. In fact, the cessation of smoking before or after the diagnosis of the disease was associated with a slower progression..$^{8}$ Results from a study done in 2012 showed that cigarette smoking increased the risk of reaching the value of six on the EDSS, in relation to other factors that have been analyzed (coffee and alcohol drinking, consumption of fish). ${ }^{39}$

Despite the above data that unambiguously showed the impact of smoking on the onset and progression of MS, it has not yet been determined exactly what would be the mechanism responsible for these results. This refers to the biochemical processes related to smoking, genetic mechanisms and influence on the development of comorbidities, which could have an impact on MS. ${ }^{10,38,40}$ Probably, there are more associated 


\section{CONCLUSION}

Although it seems reasonable to restrict or refrain from smoking in patients with MS there was no significant evidence of smoking and disease progression correlation. The individuals with a history of MS in the family, should be advised about the impact of smoking in MS, and the importance of preventing their children's exposure to passive smoking.

\section{ACKNOWLEDGEMENTS}

None.

\section{CONFLICT OF INTEREST}

None.

\section{REFERENCES}

1. Compston A, Ebers G, Lassmann H, McDonald I, Matthews B, Wekerle H. McAlpine's multiple sclerosis. 3rd ed. London: Churchill Livingstone, 1998.

2. Compston A, Coles A. Multiple sclerosis. Lancet 2002;359:1221-31.

3. Olsson T, Barcellos LF, Alfredsson L. Interactions between genetic, lifestyle and environmental risk factors for multiple sclerosis. Nat Rev Neurol 2017;13(1):2536.

4. Hedström AK. Smoking and its interaction with genetics in MS etiology. Mult Scler 2019; 25(2):180-6.

5. Antonovsky A, Leibovitz U, Smith HA, et al. Epidemiologic study of multiple sclerosis in Israel. I. An overall reviev of methods and findings. Arch Neurol 1965; 13:183-93.

6. Hernan MA, Olek MJ, Ascherio A. Cigarette smoking and incidence of multiple sclerosis. Am J Epidemiol 2001;154:69-74.

7. Hernan MA, Jick SS, Logroscino G, et al. Cigarette smoking and the progression of multiple sclerosis. Brain 2005;128:1461-5.

8. Sundstrom P, Nystrom L, Hallmans G. Smoke exposure increases the risk for multiple sclerosis. Eur $\mathrm{J}$ Neurol 2008;15:579-83.

9. Carlens C, Hergens MP, Grunewald J, et al. Smoking, use of moist snuff, and risk of chronic inflammatory diseases. Am J Respir Crit Care Med 2010;181(11):121722.

10. Hedström AK, Bäärnhielm M, Olsson T, et al. Tobacco smoking, but not Swedish snuff use, increases the risk of multiple sclerosis. Neurology 2009;73(9):696-701.

11. Salzer J, Stenlund H, Sundström P. The interaction between smoking and Epstein-Barr virus as multiple sclerosis risk factors may depend on age. Mult Scler 2014; 20(6):747-50.

12. Hedström AK, Ryner M, Fink K, et al. Smoking and risk of treatment-induced neutralizing antibodies to interferon $\beta$-1a. Mult Scler 2014;20(4):445-50.

13. Petersen ER, Søndergaard HB, Laursen JH, et al. Smoking is associated with increased disease activity during natalizumab treatment in multiple sclerosis. Mult Scler 2018;2:1352458518791753. doi: 10.1177/1352458518791753.

14. Auer M, Bsteh G, Hegen H, et al. Smoking is not associated with higher prevalence of JC virus in MS patients. Eur J Clin Microbiol Infect Dis 2018;37(5):907910. doi: 10.1007/s10096-018-3204-z.

15. Kurtzke JF. Rating neurologic instrument in multiple sclerosis: An expandede disability status scale (EDSS). Neurology 1983;33:1444-52.

16. Lalmohamed A, Bazelier MT, Van Staa TP, et al. Causes of death in patients with multiple sclerosis and matched referent subjects: A population-based cohort study. Eur J Neurol 2012;19:1007-14.

17. Khurana SR, Bamer AM, Turner AP, et al. The prevalence of overweight and obesity in veterans with multiple sclerosis. Am J Phys Med Rehabil 2009;88(2):8391.

18. Ghadirian P, Dadgostar B, Azani R, Maisonneuve P. A case-control study of the association between socio-demographic, lifestyle and medicalhistory factors and multiple sclerosis. Can J Public Health 2001;92:281-5.

19. Hawkes $\mathrm{CH}$. Smoking is a risk factor for multiple sclerosis: a metanalysis. Mult Scler 2007;13:610-5.

20. Pekmezovic T, Drulovic J, Milenkovic M, et al. Lifestyle factors and multiple sclerosis: A case-control study in Belgrade. Neuroepidemiology 2006;27(4):212-6.

21. Hedström AK. Smoking is an important risk factor for MS that overall increases the risk of the disease with approximately 50\%. Mult Scler 2018;1352458518801727. doi: $10.1177 / 1352458518801727$.

22. Hedström AK, Bäärnhielm M, Olsson T, et al. Exposure to environmental tobacco smoke is associated with increased risk for multiple sclerosis. Mult Scler 2011;17(7): 788-93.

23. Salzer J, Hallmans G, Nyström M, et al. Smoking as a risk factor for multiple sclerosis. Mult Scler 2013;19 (8):1022-7.

24. Montgomery SM, Bahmanyar S, Hillert J, et al. Maternal smoking during pregnancy and multiple sclerosis amongst offspring. Eur J Neurol 2008;15(12):1395-9.

25. Mikaeloff Y, Caridade G, Tardieu M, Suissa S. Parental smoking at home and the risk of childhood-onset multiple sclerosis in children. Brain 2007;130 (10):258995.

26. Tardieu M, Mikaeloff Y. Multiple sclerosis in children: environmental risk factors. Bull Acad Natl Med 2008;192(3):507-9.

27. Mohamed-Ali V, Goodrick S, Rawesh A, et al. Subcu- 
taneous adipose tissue releases interleukin-6, but not tumor necrosis factor-alpha, in vivo. J Clin Endocrinol Metab 1997;82:4196-200.

28. Munger K, Levin L, Hollis B, Howard N, Ascherio A. Serum 25-hydroxyvitamin D levels and risk of multiple sclerosis. JAMA 2006;296:2832-38.

29. Thacker E, Mirzaei F, Ascherio A. Infectious mononucleosis and risk for multiple sclerosis: a meta-analysis. Ann Neurol 2006;59:499-503.

30. Asadollahi S, Fakhri M, Heidari K, Zandieh A, Vafaee $\mathrm{R}$, Mansouri B. Cigarette smoking and associated risk of multiple sclerosis in the Iranian population. J Clin Neurosci 2013;20(12):1747-50.

31. Sundstrom P, Nystrom L. Smoking worsens the prognosis in multiple sclerosis. Mult Scler 2008;14:103135 .

32. Di Pauli F, Reindl M, Ehling R, et al. Smoking is a risk factor for early conversion to clinically definite multiple sclerosis. Mult Scler 2008;14:1026-30.

33. Sena A, Couderc R, Ferret-Sena V, et al. Apolipoprotein $\mathrm{E}$ polymorphism interacts with cigarette smoking in progression of multiple sclerosis. Eur J Neurol 2009;16:832-37.

34. Pittas F, Ponsonby AL, van der Mei IA, et al. Smoking is associated with progressive disease course and in- creased progression in clinical disability in a prospective cohort of people with multiple sclerosis. J Neurol 2009;256:577-85.

35. Healy BC, Ali EN, Guttmann CRG, et al. Smoking and disease progression in multiple sclerosis. Arch Neurol 2009;66:858-864.

36. Zivadinov R, Weinstock-Guttman B, Hashmi K, et al. Smoking is associated with increased lesion volumes and brain atrophy in multiple sclerosis. Neurology 2009;73:504-10.

37. Weiland TJ, Hadgkiss EJ, Jelinek GA, Pereira NG, Marck CH, van der Meer DM. The association of alcohol consumption and smoking with quality of life, disability and disease activity in an international sample of people with multiple sclerosis. J Neurol Sci 2014;15:336(1-2): 211-9.

38. Manouchehrinia A, Tench CR, Maxted J, Bibani RH, Britton J, Constantinescu CS. Tobacco smoking and disability progression in multiple sclerosis: United Kingdom cohort study. Brain 2013;136(7):2298-304.

39. D'hooghe MB, Haentjens P, Nagels G, De Keyser J. Alcohol, coffee, fish, smoking and disease progression in multiple sclerosis. Eur J Neurol 2012;19(4):616-24.

40. 40. Marie RA, Horwitz RI, Cutter G, et al. Smokers with multiple sclerosisare more likely to report co- 